\title{
Local Fixed Point Indices of Iterations of Planar Maps
}

\author{
Grzegorz Graff • Piotr Nowak-Przygodzki • \\ Francisco R. Ruiz del Portal
}

Received: 11 September 2009 / Published online: 21 January 2011

(C) Springer Science+Business Media, LLC 2011

\begin{abstract}
Let $f: U \rightarrow \mathbb{R}^{2}$ be a continuous map, where $U$ is an open subset of $\mathbb{R}^{2}$. We consider a fixed point $p$ of $f$ which is neither a sink nor a source and such that $\{p\}$ is an isolated invariant set. Under these assumption we prove, using Conley index methods and Nielsen theory, that the sequence of fixed point indices of iterations $\left\{\operatorname{ind}\left(f^{n}, p\right)\right\}_{n=1}^{\infty}$ is periodic, bounded from above by 1 , and has infinitely many non-positive terms, which is a generalization of Le Calvez and Yoccoz theorem (Annals of Math., 146, 241-293 (1997)) onto the class of non-injective maps. We apply our result to study the dynamics of continuous maps on 2-dimensional sphere.
\end{abstract}

Keywords Fixed point index $\cdot$ Conley index $\cdot$ Nielsen number $\cdot$ Periodic points $\cdot$ Iterations Mathematics Subject Classification (2000) Primary 37C25 - Secondary 37E30 • 37B30

\section{Introduction}

Let $x_{0}$ be a fixed point of a map $f$. The local fixed point index ind $\left(f, x_{0}\right) \in \mathbb{Z}$ is a topological invariant that plays important role in fixed point theory. It can also be used to study the structure of periodic points and dynamical properties of a map. In such a case the sequence of fixed point indices of iterations $\left\{\operatorname{ind}\left(f^{n}, x_{0}\right)\right\}_{n=1}^{\infty}$ may be applied. Unfortunately, it is usually

G. Graff $(\varangle) \cdot$ P. Nowak-Przygodzki

Faculty of Applied Physics and Mathematics, Gdansk University of Technology, Narutowicza 11/12, 80-233 Gdansk, Poland

e-mail: graff@mif.pg.gda.pl

P. Nowak-Przygodzki

e-mail: piotrnp@wp.pl

F. R. Ruiz del Portal

Departamento de Geometría y Topología Facultad de CC.Matemáticas,

Universidad Complutense de Madrid, 28040 Madrid, Spain

e-mail: R_Portal@mat.ucm.es 
difficult to establish the exact form of the indices for a given map. Nevertheless, during the last years the description of indices was given for many important classes of maps such as: planar homeomorphisms $[14,16,20,22]$; $\mathbb{R}^{3}$-homeomorphisms [15,23]; smooth maps $[4,9,18,24]$; and holomorphic maps $[3,6,25,26]$.

In this paper we give the restrictions for indices of some class of planar maps. The investigations of indices in dimension 2 was initiated by the famous result of Le Calvez and Yoccoz [16]. These authors considered local orientation preserving planar homeomorphism $f$ with the fixed point $p$ which is neither a sink nor a source. Under the assumption that there exists a neighborhood $V$ of $p$ such that orbits of all points (except for $p$ ) leave $V$ either in positive or negative time, they proved that the sequence of fixed point indices has very special form, namely there exist positive integers $r, q$, such that for each $n \neq 0$ :

$$
\operatorname{ind}\left(f^{n}, 0\right)= \begin{cases}1-r q & \text { if } q \mid n, \\ 1 & \text { if } \not h\end{cases}
$$

Le Calvez and Yoccoz in [16] used this fact to solve in the negative the classical Ulam problem from Scotish Book about the existence of minimal homeomorphisms of multipunctured sphere (cf. [19]).

Later Franks [7] by a use of discrete Conley index gave much shorter prove of the fact that in the sequence $\left\{\operatorname{ind}\left(f^{n}, p\right)\right\}_{n=1}^{\infty}$ there are infinitely many non-positive terms. What is more, Conley index approach turned out to be very fruitful in this problem, by this method Ruiz del Portal and Salazar reproved the formula (1.1), found the form of indices also in reversing orientation case [22] and described the behavior of $f$ near $p[20,21]$ (the reader may consult [17] for general theory of Conley index).

The aim of this paper is to generalize the result of Le Calvez and Yoccoz onto continuous maps. We dispense with the assumption that $f$ is homeomorphism, and replace the condition for orbits by the same condition for so-called solutions, which are the counterparts of full orbits for non-injective maps. Under these assumptions our main result states that $\left\{\operatorname{ind}\left(f^{n}, p\right)\right\}_{n=1}^{\infty}$ is periodic (which was conjectured by A. Szymczak in 1998 during the workshop on Conley index in Warsaw), bounded from above by 1 and has infinitely many non-positive terms (Theorem 2.4). In Sect. 6 we apply this result to examine the existence of minimal maps of two-dimensional sphere with finite number of periodic points.

The proof of our main theorem consists of two parts. In the first one we modify the map $f$ and the considered space by Conley index methods, so that we obtain a self-map $\bar{f}^{\prime}$ of a nice space with a finite number of periodic points, which coincides with $f$ near $p$. In the second step we apply the result of B. Jiang from [12] to the map $\bar{f}^{\prime}$. Jiang's theorem gives the estimates for index of each Nielsen fixed point class of $\bar{f}^{\prime}$ and for Lefschetz number. This allow us to deduce periodicity and the bounds for indices of iterations of $f$.

\section{Statement of the Main Result}

In this section we define the notions of solution and isolated invariant set and formulate the main result.

Definition 2.1 For any set $N \subset U$ define $\operatorname{Inv}^{m} N$ to be the set of $x \in N$ such that there exists an orbit segment $\left\{x_{n}\right\}_{-m}^{m} \subset N$ with $x_{0}=x$ and $f\left(x_{n}\right)=x_{n+1}$ for $n=-m, \ldots, m-1$. We will call a complete orbit containing $x$ a solution through $x$. More precisely, if $\sigma: \mathbb{Z} \rightarrow N$ is given by $\sigma(n)=x_{n}$ and $x_{0}=x$ and $f\left(x_{n}\right)=x_{n+1}$ for all $n$, we will call $\sigma$ a solution 
through $x$. We define the maximal invariant subset, $\operatorname{Inv} N$ to be $\operatorname{Inv}^{\infty} N$, the set of $x \in N$ such that there exists a solution $\sigma$ with $\{\sigma(n)\}_{-\infty}^{\infty} \subset N$ and with $\sigma(0)=x$.

Definition 2.2 A compact set $N$ is called an isolating neighborhood if $\operatorname{Inv} N \subset \operatorname{Int} N$. A set $S$ is called an isolated invariant set if there exists an isolating neighborhood $N$ with $S=\operatorname{Inv} N$.

Definition 2.3 A fixed point $p$ of a map $f$ is called a source (sink) if there is a base of compact neighborhoods $V_{\alpha}$ of $p$ such that $V_{\alpha} \subset f\left(V_{\alpha}\right)\left(f\left(V_{\alpha}\right) \subset V_{\alpha}\right)$ for every $\alpha$.

Theorem 2.4 Let $f: U \rightarrow \mathbb{R}^{2}$ be a continuous map, where $U$ is an open subset of $\mathbb{R}^{2}$. Assume that $\{p\}$ is an isolated invariant set for $f$ and $p$ is neither a sink nor a source, then

(1) $\left\{\operatorname{ind}\left(f^{n}, p\right)\right\}_{n=1}^{\infty}$ is periodic,

(2) for each $n$ ind $\left(f^{n}, p\right) \leq 1$,

(3) $\left\{\operatorname{ind}\left(f^{n}, p\right)\right\}_{n=1}^{\infty}$ has infinitely many non-positive terms.

Remark 2.5 If $f$ is an arbitrary continuous planar map, then the only restrictions on local fixed point indices are given by so-called Dold relations given in the formula $(7.1)[1,10]$.

\section{Modifying a Map by Conley Index Methods}

In this section we modify (in two steps) a map $f$ which satisfies the assumptions of Theorem 2.4 to a map $\bar{f}^{\prime}$, which is a self-map of $R$, a space which is homotopy equivalent to a bucket of circles. Furthermore $\bar{f}^{\prime}$ has finitely many periodic points and in the proximity of $p$ is equal to $f$.

Let $N$ be an isolating neighborhood.

Definition 3.1 We define the exit set of $N$ to be

$$
N^{-}:=\{x \in N: f(x) \notin \operatorname{Int} N\} .
$$

Definition 3.2 Let $S$ be an isolated invariant set and suppose $L \subset N$ is a compact pair contained in the interior of the domain of $f$. The pair $(N, L)$ is called a filtration pair for $S$ provided $N$ and $L$ are each the closures of their interiors and

(1) $\operatorname{cl}(N \backslash L)$ is an isolating neighborhood of $S$,

(2) $L$ is a neighborhood of $N^{-}$in $N$, and

(3) $f(L) \cap \operatorname{cl}(N \backslash L)=\emptyset$.

Theorem 3.3 ([8] Theorem 3.7) Let $U$ be an open subset of an n-dimensional manifold $M$ and suppose $f: U \rightarrow M$ is a continuous map with an isolated invariant set $S$. Inside any neighborhood of $S$ there exists a filtration pair $(N, L)$ such that $N$ is an $n$-dimensional manifold with boundary and $\partial L$ is an $(n-1)$-dimensional submanifold of $M$.

We consider the 2-dimensional case with $S$ equals to one fixed point, i.e. $f: U \rightarrow \mathbb{R}^{2}$ and its fixed point $p$ is an isolated invariant set. Let $N$ be an isolating neighborhood of $\{p\}$. Then, by Theorem 3.3 we get the following observation:

Remark $3.4 N$ is a closed disk with a finite numbers of holes and each component $L_{i}$ of $L$ is also a disk with some finite numbers of holes. 
For $A$, a subset of $N$, by $c c(A)$ we will denote the connected component of $A$ which contains the fixed point $p$.

Only $c c(N \backslash L)$ impacts on fixed point indices, so it would be more convenient for us to consider the sets $\tilde{L}_{i} \supset L_{i}$ instead $L_{i}$, where

$$
\tilde{L}_{i}=N \backslash \operatorname{cc}\left(N \backslash L_{i}\right) .
$$

Notice that $\partial_{N} \tilde{L_{i}}$ is a connected subset of $N$. Let $\tilde{L}$ be a union of its components: $\tilde{L}=$ $\tilde{L}_{1} \cup \cdots \cup \tilde{L}_{m}$.

Identifying each $\tilde{L}_{i}$ to a point $q_{i}$ we get a continuous map $f^{\prime}: \operatorname{cl}(N \backslash \tilde{L}) / \sim \backslash\left\{q_{1}, \ldots, q_{m}\right\} \rightarrow$ $\operatorname{cl}(N \backslash \tilde{L}) / \sim$, such that in a small neighborhood of $p, f^{\prime} \equiv f$. Now we ask whether there exists continuous extension of $f^{\prime}$ for $\left\{q_{1}, \ldots, q_{m}\right\}$.

Notice that $\partial_{N} \tilde{L}_{i} \subset \partial_{N} L_{i}$, so by Definition 3.2 (3) $f\left(\partial_{N} \tilde{L}_{i}\right) \subset \operatorname{Int} L$.

Lemma 3.5 For every $i$ there exists only one $j$ such that $f\left(\partial_{N} \tilde{L}_{i}\right) \subset \operatorname{Int} \tilde{L}_{j}$.

Proof Suppose, contrary to our claim, that $f\left(\partial_{N} \tilde{L}_{i}\right) \cap \operatorname{Int} \tilde{L}_{j} \neq \emptyset$ and $f\left(\partial_{N} \tilde{L}_{i}\right) \cap \operatorname{Int} \tilde{L}_{k} \neq \emptyset$, where $j \neq k$.

Because $\partial_{N} \tilde{L}_{i}$ is connected and by the condition (2),

$$
\partial_{N} \tilde{L}_{i} \cap N^{-}=\emptyset \text {. }
$$

Thus, $f\left(\partial_{N} \tilde{L}_{i}\right)$ is a connected set which joins $\tilde{L}_{j}$ and $\tilde{L}_{k}$ inside $\operatorname{Int} N$, so we may find a point $x_{0} \in \partial_{N} \tilde{L}_{i}$ such that

$$
f\left(x_{0}\right) \in \operatorname{Int}(N \backslash \tilde{L}) \subset N \backslash \tilde{L} \subset N \backslash L \subset \operatorname{cl}(N \backslash L),
$$

which contradicts the condition (3) of filtration pair.

Due to Lemma 3.5 there is a unique extension $\bar{f}^{\prime}$ of $f^{\prime}$

$$
\bar{f}^{\prime}: \operatorname{cl}(N \backslash \tilde{L}) / \sim \rightarrow \operatorname{cl}(N \backslash \tilde{L}) / \sim,
$$

and the continuity of $\bar{f}^{\prime}$ results from the fact that $\bar{f}^{\prime}$ is locally constant near each $q_{i}$.

On the other hand, the only periodic points which may appear for $\bar{f}^{\prime}$ are situated in $\left\{q_{1}, \ldots, q_{m}\right\}$. As a result, for every $n$, Fix $\left(\bar{f}^{\prime \prime}\right) \subset\left\{p, q_{1}, \ldots, q_{m}\right\}$. The last set consists of periodic and eventually periodic points. Without loss of generality we may assume that all of them are periodic. Then ind $\left(\bar{f}^{\prime}, q_{i}\right)=1$ if $q_{i}$ is the point of period $n$, because in a small enough neighborhood of $q_{i}$ the map $\bar{f}^{\prime \prime}$ is constantly equal to $q_{i}$.

Now for the set $R=\operatorname{cl}(N \backslash \tilde{L}) / \sim$, by Lefschetz-Hopf theorem we get:

$$
L\left(\bar{f}^{\prime n}\right)=\operatorname{ind}\left(\bar{f}^{\prime n}, R\right)=\operatorname{ind}\left(\bar{f}^{\prime \prime}, p\right)+\sum_{q_{i} \in \operatorname{Fix}\left(\bar{f}^{\prime n}\right)} \operatorname{ind}\left(\bar{f}^{\prime \prime}, q_{i}\right) .
$$

Taking into account that $\bar{f}^{\prime}$ is equal to $f^{n}$ near $p$ we obtain:

$$
\operatorname{ind}\left(f^{n}, p\right)=L\left(\bar{f}^{\prime}{ }^{n}\right)-\sum_{q_{i} \in \operatorname{Fix}\left(\bar{f}^{\prime n}\right)} \operatorname{ind}\left(\bar{f}^{\prime},{ }^{n}, q_{i}\right) .
$$

On the other hand, $H_{i}(R ; \mathbb{Q})=0$ for $i>2$, thus by definition

$$
L\left(\bar{f}^{\prime n}\right)=\sum_{k=0}^{\infty}(-1)^{k} \operatorname{tr} \bar{f}_{* k}^{\prime n}=1-\operatorname{tr} \bar{f}_{* 1}^{\prime n}+\operatorname{tr} \bar{f}_{* 2}^{\prime n},
$$


and finally:

$$
\operatorname{ind}\left(f^{n}, p\right)=1-\sum_{q_{i} \in \text { Fix }}\left(\bar{f}^{, n}\right) \text { ind }\left(\bar{f}^{\prime, n}, q_{i}\right)-\operatorname{tr} \bar{f}_{* 1}^{\prime n}+\operatorname{tr} \bar{f}_{* 2}^{\prime n} .
$$

Remark 3.6 Notice that $\sum_{q_{i} \in \mathrm{Fix}\left(\bar{f}^{\prime}{ }^{n}\right)} \operatorname{ind}\left(\bar{f}^{\prime}{ }^{n}, q_{i}\right)$ is a periodic sequence. Thus, to prove periodicity of indices we must show, by the formula (3.1), that $\left\{L\left(\bar{f}^{\prime}{ }^{n}\right)\right\}_{n}\left(\operatorname{or}\left\{-\operatorname{tr} \bar{f}^{\prime}{ }_{* 1}^{n}+\operatorname{tr} \bar{f}^{\prime}{ }_{* 2}\right\}_{n}\right.$ by (3.3)) is periodic.

\section{Jiang's Result}

Before we state Jiang's result we briefly recall the notions of Nielsen fixed point class and Nielsen number (cf. [11,13]). The set of fixed points of a map $f$ may be divided into disjoint union of fixed point classes. Two points $x$ and $y$ belong to the same class if and only if there is a path $\omega$ which joins them, such that $\omega$ and $f(\omega)$ are fixed end point homotopic. Because each fixed point class $\mathbf{F}$ is isolated in the set of fixed points of $f$, ind $(f, \mathbf{F})$ is well-defined. A fixed point class which has non-zero index is called essential. Nielsen number of $f$, denoted as $N(f)$, is a homotopy invariant equal to the number of essential fixed points classes of $f$ and gives lower bound for the number of fixed points in the homotopy class of $f$.

The following theorem was proved for surfaces by B. Jiang in [12].

Theorem 4.1 Let $X$ be a connected compact surface with Euler characteristic $\chi(X)<0$, and suppose $f: X \rightarrow X$ is a continuous map. Then:

(A) for every fixed point class $\mathbf{F}$ of $f$ ind $(f, \mathbf{F}) \leq 1$.

(B) $|L(f)-\chi(X)| \leq N(f)-\chi(X)$.

In the proof of our main theorem we will also need the following lemma of Babenko and Bogatyi (Theorem 2.2 in [1]):

Lemma 4.2 If the sequence of Lefschetz numbers of iterations $\left\{L\left(f^{n}\right)\right\}_{n=1}^{\infty}$ is bounded, then it is periodic.

\section{Proof of Theorem 2.4}

The fixed point $p$ is neither a sink nor a source, so we choose its small neighborhood $V \subset U$, such that for any compact neighborhood $W \subset V$

$$
\text { neither } f(W) \subset W \text { nor } f(W) \supset W \text {. }
$$

Next, by Theorem 3.3 we find inside $V$ a filtration pair $(N, L)$ for which we apply the identification described in Sect. 3, obtaining the space $R$ and its self-map $\bar{f}^{\prime}$.

We prove Theorem 2.4 considering some cases and their subcases.

Case (I) None of $\tilde{L_{i}}$ forms an annulus around $p$.

Under the above assumption the second homology group of $R$ disappear, and thus $\operatorname{tr} \bar{f}^{\prime \prime}{ }_{* 2}=$ 0 in the formula (3.3). Notice that $R$ is also the disk with holes and the number of holes in $R$ is not greater than the numbers of holes in $N$ (cf. Remark 3.4 and the definition of $R$ ).

Now, for the three following subcases we prove part (1) and (2) of Theorem 2.4.

Case (Ia) There are no holes in $R$. Then, in the formula (3.3) $\operatorname{tr} \bar{f}^{\prime}{ }_{* 1}^{n}=0$, so by Remark 3.6 the sequence of indices is periodic. On the other hand, let us remind that if $q_{i}$ is the point of period $n$ for $\bar{f}^{\prime}$ then

$$
\operatorname{ind}\left(\bar{f}^{\prime n}, q_{i}\right)=1 \text {, }
$$


so $\sum_{q_{i} \in \text { Fix }\left(\bar{f}^{\prime n}\right)} \operatorname{ind}\left(\bar{f}^{\prime \prime}, q_{i}\right) \geq 0$. Thus, again by the formula (3.3), we get immediately the needed bound for ind $\left(f^{n}, p\right)$.

Case (Ib) There is one hole in $R$. Let us denote by $D$ the hole in $R$, and by $d \in \mathbb{Z}$ the number such that $\bar{f}^{\prime}{ }_{* 1}(\alpha)=d \alpha$, where $\alpha=\partial D$ is a generator of $H_{1}(R ; \mathbb{Q})$.

Notice that $\bar{f}^{\prime}$ has only finite number of periodic points, thus $\left\{N\left(\bar{f}^{\prime},\right\}_{n}\right.$ is bounded. On the other hand, $\bar{f}^{\prime}$ is homotopy equivalent to a self-map $g$ of a circle with degree $d$. Homotopy equivalent maps have the same Nielsen and Lefschetz numbers. Furthermore, the modulus of Lefschetz number is equal to Nielsen number for self-maps of the circle, thus we obtain:

$$
\left|L\left(\bar{f}^{\prime \prime}\right)\right|=\left|L\left(g^{n}\right)\right|=N\left(g^{n}\right)=N\left(\bar{f}^{\prime n}\right),
$$

so $\left\{L\left(\bar{f}^{\prime}{ }^{n}\right)\right\}_{n=1}^{\infty}$ is bounded, and thus by Lemma 4.2 it is periodic. Finally, by Remark 3.6 we get the periodicity of indices.

To prove part (2) of Theorem 2.4, we use the formula (3.3). Notice that $\operatorname{tr} \bar{f}_{* 1}^{\prime n}=d^{n}$. On the other hand, as Lefschetz numbers $L\left(f^{n}\right)=1-d^{n}$ are bounded, we get that $d \in\{-1,0,1\}$.

The formula (3.3) takes then the form:

$$
\operatorname{ind}\left(f^{n}, p\right)=1-\sum_{q_{i} \in \operatorname{Fix}\left(\bar{f}^{\prime},{ }^{n}\right)} \operatorname{ind}\left(\bar{f}^{\prime \prime}, q_{i}\right)-d^{n} .
$$

As a result, if $d \in\{0,1\}$ then by (5.2) ind $\left(f^{n}, p\right) \leq 1$. Let us consider the case of $d=-1$. Then, by (5.3) $N\left(\bar{f}^{\prime}\right)=|1-d|=2$, so $\bar{f}^{\prime}$ has at least two fixed points. One of them must be $p$, let us denote the other one as $q$. Then:

$$
\operatorname{ind}\left(f^{n}, p\right) \leq 1-\operatorname{ind}\left(\bar{f}^{\prime}{ }^{n}, q\right)-(-1)^{n} \leq 1 .
$$

Case (Ic) There are at least two holes in $R$. Theorem 4.1, as it is stated, acts for surfaces, but from the proof ([12] Lemma B p. 476) it follows that it is valid for a space which is homotopy equivalent to a graph. As this situation holds in our case - $R$ is homotopy equivalent to a graph with the same Euler characteristic and $\chi(R)<0$, the thesis of Theorem 4.1 holds for $R$.

We remind that

$$
\text { Fix }\left(\bar{f}^{\prime}{ }^{n}\right) \subset\left\{p, q_{1}, \ldots, q_{m}\right\} .
$$

Again, because there is only finite number of periodic points of $\bar{f}^{\prime},\left\{N\left(\bar{f}^{\prime},\right\}_{k}\right.$ is bounded. As a result, by Theorem 4.1 (B) $\left\{L\left(\bar{f}^{\prime}{ }^{k}\right)\right\}_{k}$ is also bounded and thus by Lemma 4.2 and Remark 3.6 the sequence of indices is periodic.

Now we prove part (2) of Theorem 2.4. Let us denote by $\mathbf{F}_{p}^{n}$ the fixed point class of $\bar{f}^{\prime} n$ which contains $p$. Then $\mathbf{F}_{p}^{n}=\left\{p, q_{1}, \ldots, q_{s}\right\}$, where $0 \leq s \leq m$.

By the equality (5.2) we get:

$$
\operatorname{ind}\left(\bar{f}^{\prime \prime},\left\{q_{1}, \ldots, q_{s}\right\}\right)=s .
$$

Now let us observe that ind $\left(\bar{f}^{\prime}{ }^{n}, \mathbf{F}_{p}^{n}\right) \leq 1$ by Theorem 4.1 (A). Thus, due to the equality (5.6) and the additivity of fixed point index, we get that

$$
\operatorname{ind}\left(f^{n}, p\right)=\operatorname{ind}\left(\bar{f}^{\prime n}, p\right) \leq 1-s \leq 1 .
$$

Now we prove part (3). We use the formula (3.3). Remind that $\operatorname{tr} \bar{f}_{* 2}^{\prime n}=0$. On the other hand, $\operatorname{tr} \bar{f}^{\prime}{ }_{* 1}^{n}=\operatorname{tr} A^{n}$, where $A$ is some integral matrix. If $A$ is nilpotent, then $\operatorname{tr} A^{n}=0$ for all $n$, otherwise $\operatorname{tr} A^{n}>0$ for infinitely many $n$ [7], so in the second case the thesis is proved. Assume that $\operatorname{tr} \bar{f}_{* 1}^{\prime n}=0$ for all $n$, then if there exist some periodic point $q_{i}$ the theorem holds. 
The only case we have to check is when there are no periodic points, so $L=\emptyset$. But this case is impossible, because then $f(N) \subset N$, which contradicts (5.1).

This completes the proof in Case I.

Case (II) Some of $\tilde{L}_{i}$ forms an annulus around $p$.

Assume that $\tilde{L}_{1}$ is the closest to $p$ of all $\tilde{L}_{i}$ that form an annulus around $p$. Let us remind that $\tilde{L}_{1}=N \backslash \operatorname{cc}\left(N \backslash L_{1}\right)$. Denote by $D$ the open disk bounded by the annulus $\tilde{L}_{1}$, let $\tilde{L}_{2}, \ldots, \tilde{L}_{k}$ be the set of all $\tilde{L}_{i}$ contained in $D$.

Case (IIa) There are no holes in $R$. In this case we get that $H_{1}(R ; \mathbb{Q})=0$, and $\bar{f}^{\prime}$ is a self-map of 2-dimensional sphere. Thus, $L\left(\bar{f}^{\prime}\right)=1+d^{n}$, where $d$ is the degree of $\bar{f}^{\prime}$, and the formula (3.3) takes the form:

$$
\operatorname{ind}\left(f^{n}, p\right)=1+d^{n}-\sum_{q_{i} \in \operatorname{Fix}\left(\bar{f}^{\prime}{ }^{n}\right)} \operatorname{ind}\left(\bar{f}^{\prime}{ }^{n}, q_{i}\right) .
$$

Let us consider the following compact neighborhood of $p: W=\bar{D} \backslash \bigcup_{i=2}^{k} \operatorname{Int}\left(\tilde{L}_{i}\right)$. As $p$ is not a source, by (5.1) $W \not \subset f(W)$. This implies that there exists $q \in W$ such that $q \notin f(W)$. In fact, by the compactness of $W$ we may choose $q$ in the interior of $W$. As a consequence, the map $\bar{f}^{\prime}: S^{2} \rightarrow S^{2}$ is not onto, so $d=0$. Finally, indices are bounded from above by 1 and by Remark 3.6 periodic. Because there is at least one periodic point of $\bar{f}^{\prime}$ (i.e. $\tilde{L}_{1}$ after identification to a point), there must be infinitely many non-positive terms in $\left\{\operatorname{ind}\left(f^{n}, p\right)\right\}_{n=1}^{\infty}$.

Case (IIb) There are some holes in $R$. Then the situation reduces to the Case I, which completes the proof of the whole theorem.

\section{Existence of Minimal Maps on Two-dimensional Sphere}

The classical problem posed by Ulam (Problem 115 in [19]) asked whether there exists minimal homeomorphism of multipunctured two-dimensional sphere, or in other words whether there is a homeomorphism $f$ of $\mathbb{S}^{2} \backslash\left\{p_{1}, \ldots, p_{r}\right\}$, such that the full orbit of every point $x,\left\{f^{n}(x)\right\}_{n=-\infty}^{n=+\infty}$, is dense. Le Calvez and Yoccoz, using the formula (1.1) proved that there are no such homeomorphisms.

We will consider the counterpart of Ulam problem for surjective maps which may not be injective, namely for a class of surjective continuous self-maps of $S^{2}$ with finite number of periodic points, denoted as PerFin.

First of all, let us notice that there are a few alternative ways of expressing the Ulam question for non-injective maps. We state below three versions of it. We ask whether there exists a map $f \in$ PerFin such that:

(I) $\forall_{x \in S^{2} \backslash \operatorname{Per}(f)}$ each solution through $x$ is dense.

(II) $\forall x \in S^{2} \backslash \operatorname{Per}(f)$ at least one solution through $x$ is dense.

(III) $\forall_{x \in S^{2} \backslash \operatorname{Per}(f)}$ the sum of all solutions through $x$ is dense.

Notice that surjectivity of $f$ implies that $\forall_{x \in S^{2} \backslash \operatorname{Per}(f)}$ there exists a solution through $x$.

It is obvious that the following implications hold:

$$
\text { (I) } \Rightarrow \text { (II) } \Rightarrow \text { (III) }
$$

We will demonstrate that for every map in PerFin (I) is false (Theorem 6.1) but there are some maps in PerFin for which (II) (and thus also (III)) hold (Theorem 6.5).

We start with showing non-existence of a map satisfying (I). Although the general line of reasoning in the proof is similar to the one used in [16], we must use much more subtle arguments than those which are valid for homeomorphisms. 
Theorem 6.1 There is no $f \in$ PerFin such that each solution through each point $x \in S^{2} \backslash$ $\operatorname{Per}(f)$ is dense.

Proof Conversely, suppose that

(*) for some map $f$ each solution through each point $x \in S^{2} \backslash \operatorname{Per}(f)$ is dense.

Let $G$ be the set of all periodic points of $f, G=\left\{p_{1}, \ldots, p_{r}\right\}$. Let us take even $k$ such that all points in $G$ are fixed for $f^{k}$. Then, consider $h=f^{k}$, the self-map of $S^{2}$.

First of all we show that:

(1) each $\left\{p_{i}\right\}$ is an isolated invariant set for $h$,

(2) none of $p_{i}$ is

(2a) a sink for $h$,

(2b) a source for $h$.

To prove (1) assume contrary to our claim that for some point, say $p_{1}$, in every neighborhood of $p_{1}$ there is a solution for $h$ different from $\left\{p_{1}\right\}$.

For each $i=1, \ldots, r$ we consider $W_{i}$, a small compact neighborhood of $p_{i}$, such that $W_{l} \cap W_{s}=\emptyset$ for $l \neq s$. Then the sum $\bigcup_{i=1}^{r} W_{i}$ do not cover $S^{2}$. For each $i=1, \ldots, r$ we choose $W_{i}^{\prime} \subset W_{i}$, a compact neighborhood of $p_{i}$ such that

$$
\forall_{1 \leq t \leq k-1} f^{t}\left(W_{i}^{\prime}\right) \subset \bigcup_{i=1}^{r} W_{i} .
$$

We find a solution for $h$ contained in $W_{1}^{\prime}$ which goes through some point $y$. Then the solution through $y$ for $f$ must be contained in $\bigcup_{i=1}^{r} W_{i}$, so is not dense, contradiction with (*).

Now we will prove (2). In this part of the proof we will need the following two facts proved in [8] and [2].

Lemma 6.2 ([8] Proposition 3.2) Let $G$ be an isolated invariant set for $f$ and $W$ be its isolated neighborhood. Let us take an $x \in W$ and consider $\left\{x_{n}\right\}_{-\infty}^{+\infty}-$ a solution through $x$. If for all $n \geq 0 x_{n} \in W$, then $\omega(x) \subset G$; if for all $n \leq 0 x_{n} \in W$, then $\alpha(x) \subset G$.

Lemma 6.3 ([2] Theorem 5) Let $X$ be a compact Hausdorff space without isolated points. Let $g: X \rightarrow X$ be a continuous map and let $D_{g}$ denote the set of points $x \in X$ whose forward orbit $\left\{x_{n}\right\}_{n=0}^{+\infty}$ is dense. Then either $D_{g}=X$ or $\operatorname{Int}_{X}\left(D_{g}\right)=\emptyset$.

(2a) Again, contrary to our claim, assume that there is $p_{1} \in G$ which is a sink for $h$. Let us notice that $\bigcup_{i=1}^{r} W_{i}$, which was defined in the proof of part (1), constitutes an isolating neighborhood of $G$ for the map $f$. For $p_{1}$ let us choose its compact neighborhood $V_{1} \subset W_{1}^{\prime}$ such that $h\left(V_{1}\right) \subset V_{1}$. Then for each $y \in V_{1}$ and non-negative $n$ there is:

$$
f^{n}(y) \in \bigcup_{i=1}^{r} W_{i}=W .
$$

Consider now $x \in V_{1}$, which is different from $p_{1}$. By (6.1) $f^{n}(x) \in W$ for all $n \geq 0$, so by Lemma $6.2 \omega(x) \subset G$. As $G$ is finite, the solution through $x$ is dense if and only if $\left\{x_{n}\right\}_{n=-\infty}^{0}$ is dense. On the other hand, in such situation there exist $p>q>0$, such that $x_{-q} \notin W$ and $x_{-p} \in V_{1}$. The last statement imply however that $f^{p-q}\left(x_{-p}\right)=x_{-q} \notin W$, but by (6.1) $f^{p-q}\left(x_{-p}\right) \in W$, contradiction proves part (2a).

(2b) We use the notation from the proof of part (1). To obtain a contradiction suppose that there is $p_{1} \in G$ which is a source. Let us choose a compact neighborhood $Z_{1} \subset W_{1}^{\prime}$ such 
that $Z_{1} \subset h\left(Z_{1}\right)$. Then for each $y \in Z_{1} \backslash\left\{p_{1}\right\}$ there is a negative semi-solution through $y$ for $h$, which is contained in $Z_{1}$. As a result, there is a negative semi-solution through $y$ for $f$, which is contained in $W$. Then, by Lemma $6.2 \alpha(y) \subset G$. As $G$ is finite, the solution through $y$ for $f$ is dense if and only if the positive semi-orbit for $f,\left\{y_{n}\right\}_{n=0}^{\infty}$, is dense. Notice that this property should be satisfied for every $y \in Z_{1} \backslash\left\{p_{1}\right\}$, thus the set $D_{f}$ (defined in Lemma 6.3 for $g=f$ and $X=S^{2}$ ) has not empty interior. On the other hand, $D_{f} \neq S^{2}$ because $p_{1} \notin D_{f}$. We get the contradiction with the thesis of Lemma 6.3, which proves (2b).

Now we are in a position to prove the theorem. We get: $L\left(h^{j}\right)=1+d^{j}$, where $d$ is the degree of $h$. On the other hand, by Lefschetz-Hopf theorem, for each $j>0$ :

$$
L\left(h^{j}\right)=\sum_{i=1}^{r} \operatorname{ind}\left(h^{j}, p_{i}\right) .
$$

Thus, by Theorem 2.4 Lefschetz numbers are bounded. As a consequence ( $h=f^{k}$ and $k$ is even), $d \in\{0,1\}$, which implies $L\left(h^{j}\right) \in\{1,2\}$. Now we show that the sequence in the right hand-side of the formula (6.2) has infinitely many non-positive terms, which will give us the contradiction. We apply the argument of Franks used in [7]. Let us take the index pair for $\left\{p_{i}\right\}$, which is an isolated invariant set of $h$. Then, identify every $\tilde{L}_{i}$ to one point $q$ (instead to different ones $q_{i}$ as we did in Sect. 3). This point is a sink and has indices of iterations equal to one. Under such identification the counterpart of the formula (3.3) for $h$ takes the form:

$$
\operatorname{ind}\left(h^{j}, p_{i}\right)=-\operatorname{tr} A_{i}^{j},
$$

where $A_{i}$ is some integral matrix corresponding to the first homology group (the trace of the induced map on the second homology group disappear by the same arguments as used in Case II).

Now consider the matrix $A=\bigoplus_{i=1}^{r} A_{i}$. We get:

$$
\sum_{i=1}^{r} \operatorname{ind}\left(h^{j}, p_{i}\right)=\sum_{i=1}^{r}-\operatorname{tr} A_{i}^{j}=-\operatorname{tr} A^{j} .
$$

On the other hand, $\operatorname{tr} A^{j} \geq 0$ for infinitely many $j>0$ [7], which proves that in the sequence $\left\{\sum_{i=1}^{r} \operatorname{ind}\left(h^{j}, p_{i}\right)\right\}_{j}$ there are infinitely many non-positive terms. This gives the contradiction with the formula (6.2) and completes the proof.

Now we construct a map satisfying the condition (II).

Let us consider the standard tent map: $T:[0,1] \rightarrow[0,1]$, given by the formula:

$$
T(x)= \begin{cases}2 x & \text { if } x \in\left[0, \frac{1}{2}\right], \\ 2(1-x) & \text { if } x \in\left[\frac{1}{2}, 1\right] .\end{cases}
$$

Notice that:

$$
\forall_{x \in[0,1]} \forall_{\Delta} \exists_{N} \forall_{n \geq N} \exists_{t \in \Delta} \quad T^{n}(t)=x,
$$

where $\Delta$ denotes any subinterval of $[0,1]$.

Lemma 6.4 Through each point $x \in[0,1]$ goes at least one solution for $T$ which is dense.

Proof Let $x$ and $\Delta$ be fixed and $t$ found by (6.5) for some $n$. Assume that $\Delta^{\prime}$ is another subinterval of $[0,1]$. Then by (6.5) one may find natural $n^{\prime}$ and $t^{\prime} \in \Delta^{\prime}$ such that $T^{n^{\prime}}\left(t^{\prime}\right)=t$.

In this way we may inductively (taking smaller and smaller covers of $[0,1]$ ) find in every subinterval an element of the negative semi-solution through $x$. 
Theorem 6.5 There exists $F \in$ PerFin such that through each point $x \in S^{2} \backslash \operatorname{Per}(f)$ goes at least one solution which is dense.

Proof We define the map $F: S^{2} \rightarrow S^{2}$ in the following way. We will represent the twodimensional sphere $S^{2}$ as the suspension of $S^{1}$, i.e. $S^{2}=\left(S^{1} \times[0,1]\right) / \sim$, where $S^{1} \times\{0\} \sim$ $*, S^{1} \times\{1\} \sim *$, are contracted to $s=\left[S^{1} \times 0\right]$ and $n=\left[S^{1} \times 1\right]$ South and North Pole, respectively. We define a map

$$
F([\alpha, x])=[(O(\alpha), T(x))],
$$

where $O: S^{1} \rightarrow S^{1}$ is an irrational rotation. Notice that $\operatorname{Per}(F)=\{s\}$.

Let $\Delta_{1} \subset S^{1}, \Delta_{2} \subset[0,1]$ be arbitrary small closed intervals. Let us consider $\Gamma=$ $\left(\Delta_{1} \times \Delta_{2}\right) / \sim$, a subset of $S^{2}$.

We will prove that

$$
\forall_{[\alpha, x] \in S^{2} \backslash\{s\}} \forall_{\Gamma} \exists_{n} \exists_{\left[\alpha^{\prime}, t\right] \in \Gamma} \quad F^{n}\left(\left[\alpha^{\prime}, t\right]\right)=[\alpha, x] .
$$

Indeed, for a given $x$ and $\Delta_{2}$ we find $N$ satisfying (6.5). Next, there exists $n>N$ such that $O^{n}\left(\alpha^{\prime}\right)=\alpha$ for some $\alpha^{\prime} \in \Delta_{1}$. Finally, by (6.5) we choose $t \in \Delta_{2}$ such that $T^{n}(t)=x$.

Now, (6.6) implies, in the same manner as in the proof of Lemma 6.4, that through each point $[\alpha, x] \in S^{2} \backslash\{s\}$ goes at least one negative semi-solution for $F$ which is dense.

\section{Final Remarks}

Our main Theorem 2.4 provides strong restrictions for sequences of indices of iterations. An interesting question is whether there are further bounds for the sequence $\left\{\operatorname{ind}\left(f^{n}, p\right)\right\}_{n=1}^{\infty}$ for $f$ belonging to the considered class of planar maps.

In 1984 Albrecht Dold proved that every sequence of indices must satisfy some congruences [5], called Dold relations :

$$
\sum_{k \mid n} \mu(n / k) \operatorname{ind}\left(f^{k}, p\right) \equiv 0 \quad(\bmod n),
$$

where $\mu$ is the classical Möbius function, i.e., $\mu: \mathbb{N} \rightarrow \mathbb{Z}$ is defined by the following three properties: $\mu(1)=1, \mu(k)=(-1)^{s}$ if $k$ is a product of $s$ different primes, $\mu(k)=0$ otherwise.

Notice that the conditions (1)-(3) of Theorem 2.4 does not imply Dold relations. As a consequence, the formula (7.1) gives the further bounds for indices. For example, the sequence $(0,1,0,1, \ldots)$ satisfies all three conditions, but cannot be a sequence of local indices of iterations of any map $f$, because Dold relations do not hold: $1=\operatorname{ind}\left(f^{2}, p\right) \not \equiv 0(\bmod 2)$. The above discussion lead us to the following conjecture.

Problem. Assume that $\{p\}$ is an isolated invariant set for a planar map $f$ and $p$ is neither a sink nor a source. Is that true that all restrictions for $\left\{\operatorname{ind}\left(f^{n}, p\right)\right\}_{n=1}^{\infty}$ are given by Dold relations and the conditions (1)-(3) of Theorem 2.4 ?

Remark 7.1 The proof of our main result i.e. Theorem 2.4 is based on topological properties of the plane and cannot be generalized for higher dimensions. However, it has been recently shown, by the application of Conley index and topological entropy, that $\left\{\operatorname{ind}\left(f^{n}, p\right)\right\}_{n=1}^{\infty}$ is periodic for $\mathbb{R}^{3}$-homeomorphism $f$ for which $\{p\}$ is an isolated invariant set [15]. 
Acknowledgements The authors thank Professor Xuezhi Zhao for drawing their attention to the Jiang's paper [12]. The research of two first authors was supported by Polish National Grant No. N N201 373236. The third author has been supported by MICINN, MTM 2009-07030.

\section{References}

1. Babenko, I.K., Bogatyi, S.A.: The behavior of the index of periodic points under iterations of a mapping. Math. USSR Izv. 38, 1-26 (1992)

2. Bernardes, N.: On the set of points with a dense orbit. Proc. Amer. Math. Soc. 128(11), 3421-3423 (2000)

3. Bogatyi, S.A.: Local indices of iterations of a holomorphic mapping. (Russian) General topology. Spaces and mappings, 48-61, Moskov. Gos. Univ., Moscow, (1989)

4. Chow, S.N., Mallet-Parret, J., Yorke, J.A.: A periodic point index which is a bifurcation invariant, Geometric dynamics (Rio de Janeiro, 1981), 109-131, Springer Lecture Notes in Math. 1007, Berlin (1983)

5. Dold, A.: Fixed point indices of iterated maps. Invent. Math. 74, 419-435 (1983)

6. Fagella, N., Llibre, J.: Periodic points of holomorphic maps via Lefschetz numbers. Trans. Amer. Math. Soc. 352(10), 4711-4730 (2000)

7. Franks, J.: The Conley index and non-existence of minimal homeomorphisms. Illinois J. Math. 43(3), 457-464 (1999)

8. Franks, J., Richeson, D.: Shift equivalence and the Conley index. Trans. Amer. Math. Soc. 352(7), 3305-3322 (2000)

9. Graff, G., Nowak-Przygodzki, P.: Fixed point indices of iterations of $C^{1}$ maps in $\mathbb{R}^{3}$. Discrete Cont. Dyn. Syst. 16(4), 843-856 (2006)

10. Graff, G., Nowak-Przygodzki, P.: Sequences of fixed point indices of iterations in dimension 2. Univ. Iagel. Acta Math. XLI, 135-140 (2003)

11. Jezierski, J., Marzantowicz, W.: Homotopy methods in topological fixed and periodic points theory, topological fixed point theory and its applications, 3. Springer, Dordrecht (2005)

12. Jiang, B.J.: Bounds for fixed points on surfaces. Math. Ann. 311(3), 467-479 (1998)

13. Jiang, B.J.: Lectures on the Nielsen fixed point theory, Contemp. Math. 14, Amer. Math. Soc., Providence (1983)

14. Le Calvez, P.: Dynamique des homomorphismes du plan au voisinage d'un point fixe. Ann. Sci. École Norm. Sup. (4) 36( 1), 139-171 (2003)

15. Le Calvez, P., Salazar, J.M., Ruiz del Portal, F.R.: Fixed point indices of the iterates of $\mathbb{R}^{3}$-homeomorphisms at fixed points which are isolated invariant sets. J. London Math. Soc. 82(2), 683-696 (2010)

16. Le Calvez, P., Yoccoz, J.-C.: Un théoreme d'indice pour les homéomorphismes du plan au voisinage d'un point fixe. Annals of Math. 146, 241-293 (1997)

17. Mischaikow, K., Mrozek, M.: Conley index. Handbook of dynamical systems, vol. 2. pp. 393-460. North-Holland, Amsterdam (2002)

18. Marzantowicz, W., Przygodzki, P.: Finding periodic points of a map by use of a $k$-adic expansion. Discrete Contin. Dyn. Syst. 5, 495-514 (1999)

19. Mauldin, R.D. (red): The Scottish book, Birkhäuser, Boston (1981)

20. Ruiz del Portal, F.R., Salazar, J.M.: A Poincaré formula for the fixed point indices of the iterates of arbitrary planar homeomorphisms, Fixed Point Theory Appl. Art. ID 323069, pp. 31 (2010)

21. Ruiz del Portal, F.R., Salazar, J.M.: A stable/unstable manifold theorem for local homeomorphisms of the plane. Ergodic Theory Dynam. Systems 25(1), 301-317 (2005)

22. Ruiz del Portal, F.R., Salazar, J.M.: Fixed point index of iterations of local homeomorphisms of the plane: a Conley index approach. Topology 41(6), 1199-1212 (2002)

23. Ruiz del Portal, F.R., Salazar, J.M.: Indices of the iterates of $\mathbb{R}^{3}$-homeomorphisms at Lyapunov stable fixed points. J. Diff. Eq. 244(5), 1141-1156 (2008)

24. Shub, M., Sullivan, P.: A remark on the Lefschetz fixed point formula for differentiable maps. Topology 13, 189-191 (1974)

25. Zhang, G.Y.: Fixed point indices and invariant periodic sets of holomorphic systems. Proc. Amer. Math. Soc. 135(3), 767-776 (2007) (electronic)

26. Zhang, G.Y.: Fixed point indices and periodic points of holomorphic mappings. Math. Ann. 337(2), 401-433 (2007) 